\title{
APPLICATION OF GENETIC ALGORITHM IN THE MODELING OF LEAF CHLOROPHYLL LEVEL BASED ON VIS/NIR REFLECTION SPECTROSCOPY
}

\author{
Haiqing Yang ${ }^{1,2}$,Yong $\mathrm{He}^{1, *}$ \\ ${ }^{1}$ College of Biosystems Engineering and Food Science, Zhejiang University, Hangzhou, \\ Zhejiang Province, P. R. China 310029 \\ 2 College of Information Engineering, Zhejiang University of Technology, Hangzhou, \\ Zhejiang Province, P. R. China 310032 \\ * Corresponding author, Address: 268 kaixuan RD. Hangzhou 310029, P. R. China, Tel: +86- \\ 571-86971143, Fax:+86-571-86971143,Email: yhe@zju.edu.cn
}

Abstract: In order to detect leaf chlorophyll level nondestructively and instantly, VIS/NIR reflection spectroscopy technique was examined. In the test, 70 leaf samples were collected for model calibration and another 50 for model verification. Each leaf sample was optically measured by USB4000, a modular spectrometer. By the observation of spectral curves, the spectral range between $650 \mathrm{~nm}$ and $750 \mathrm{~nm}$ was found significant for mathematic modeling of leaf chlorophyll level. SPAD-502 meter was used for chemometrical measurement of leaf chlorophyll value. In the test, it was found necessary to put leaf thickness into consideration. The procedure of shaping the prediction model is as follows: First, leaf chlorophyll level prediction equation was created with uncertain parameters. Second, a genetic algorithm was programmed by Visual Basic 6.0 for parameter optimization. As the result of the calculation, the optimal spectral range was narrowed within $683.24 \mathrm{~nm}$ and $733.91 \mathrm{~nm}$. Compared with the $\mathrm{R} 2=0.2309$ for calibration set and $\mathrm{R} 2=0.5675$ for verification set without concerns of leaf thickness, the effect of leaf thickness on the spectral modeling is significant: the R2 of calibration set and verification set has been improved as high as 0.8658 and 0.9161 respectively. The test showed that it is practical to use VIS/NIR reflection spectrometer for the quantitative determination of leaf chlorophyll level.

Keywords: leaf chlorophyll level, VIS/NIR reflection spectroscopy, genetic algorithm, leaf thickness

Please use the following format when citing this chapter:

Yang, H. and He, Y., 2009, in IFIP International Federation for Information Processing, Volume 293, Computer and Computing Technologies in Agriculture II, Volume 1, eds. D. Li, Z. Chunjiang, (Boston: Springer), pp. 179-188. 


\section{INTRODUCTION}

The evaluation of leaf chlorophyll level is essential for farmers to judge the growing conditions of crops they plant. One of convenient ways to detect leaf chlorophyll is to use SPAD chlorophyll meter. SPAD is the short name of Soil Plant Analysis Development. Minolta Camera, Japan, is the wellknown manufacturer in the world producing a serial of SPAD meters. Basically, SPAD meter measures the relevant ratio of the absorbance intensities of two bands of red light and near infrared light, and exhibits not the real chlorophyll level but the "greenness degree" of the leaf it detects(Minolta Camera Co. Ltd, 1989). It is also widely used to measure the nitrogen level of plant so as to indirectly help farmers learn about whether the growing crop is lack of nitrogen(Wu et al., 1998; Chang et al., 2003; Lopez-Bellido et al., 2004; Zhu Zheyan et al., 2006; Qiu Zhengjun et al., 2007; Hoel et al., 1998). In this way, the fertilizer can be exactly managed with the purpose of protecting ambient pollution, especially the water sources around. As for remotely monitoring the growth conditions of crops, however, SPAD meter shows its deficiencies, such as mechanical touching with the leave sample, shortage of other measurements like $\mathrm{pH}$, water content, etc. Nowadays, a few of researches have been conducted to use other techniques to detect the growing conditions of plant(Cho et al., 2007; Pinkard et al., 2006; Wu et al., 2007). Thanks to its fast signal detection and advance chemometrical methods, visual and near infrared spectroscopy (Vis/NIRS) becomes one of promising techniques in the field of plant and soil information detection(Zhu Zheyan et al., 2006; Qiu Zhengjun et al., 2007). Bauerle et al.(2004) pointed out that in the spectral range from $400 \mathrm{~nm}$ to $700 \mathrm{~nm}$ there existed some links between the readings of SPAD meter and the transmission spectra of leaves. But he also emphasized that no obvious relationship can be established between leaf SPAD measurement and its reflection spectrum. Thus, some effort should be made to explore the problem. In this study, we use fiber-optic OEM modular spectrometer to build the mathematical model of leaf chlorophyll level based on Vis/NIR reflection spectroscopy.

\section{MATERIALS AND METHODS}

\subsection{Leaf samples and instruments}

All leaf samples were collected from Agriculture Campus, Zhejiang University, in two periods of time. On January 15, 2008, 50 leaves were 
collected and 70 measuring points were selected as the calibration set. On January 25, 2008, another 40 leaves were picked with 50 sample points as the verification set. All leaf samples were water-cleaned and natural air-dried under room temperature of $25^{\circ} \mathrm{C}$. Each measuring point is ink-marked by a circle with $1 \mathrm{~cm}$ diameter and given a sequence number. The circle center is used for measuring point. In the test, a USB4000 fiber-optic OEM modular spectrometer (Ocean Optics, USA), with its spectral range from $346.01 \mathrm{~nm}$ to $1038.08 \mathrm{~nm}$, is used for spectral collection. Its integration time can be adjusted from $3.8 \mathrm{~ms}$ to $10 \mathrm{~s}$. A reflective fiber-optic is vertically fixed about $5 \mathrm{~cm}$ high above the surface of leaf to be tested. After the spectroscopic measurement, each leaf sample is measured by SPAD-502 meter (Minolta Camera, Japan) for its leaf chlorophyll level. Data processing program is written by Visual Basic 6.0 and spectral curves are drawn in Matlab 7.0 and Microsoft Excel 2003. Leaf thickness is measured by a micrometer (Shanghai Measuring Meter Factory, China) with its precision $0.01 \mathrm{~mm}$.

\subsection{Spectral data preprocessing}

The original reflective spectra of leaf samples are drawn in Fig.1. Dark spectrum refers to the one with illumination light switched off; reference spectrum means the reflective spectrum of illumination light itself. Due to the existence of dark spectrum, each sample spectrum has a deviation. Let $D(\lambda), R(\lambda)$ and $S(\lambda)$ the intensities of dark spectrum, reference spectrum and sample spectrum at wavelength $\lambda$. To eliminate the influence of dark spectrum on reference spectrum and sample spectrum, we design spectral reflection rate of sample as follows,

$$
R E F(\lambda)=\frac{S(\lambda)-D(\lambda)}{R(\lambda)-D(\lambda)}
$$

Then, the spectral absorbance rate of sample can be calculated by

$$
A B S(\lambda)=-\log _{10}(\operatorname{REF}(\lambda))
$$




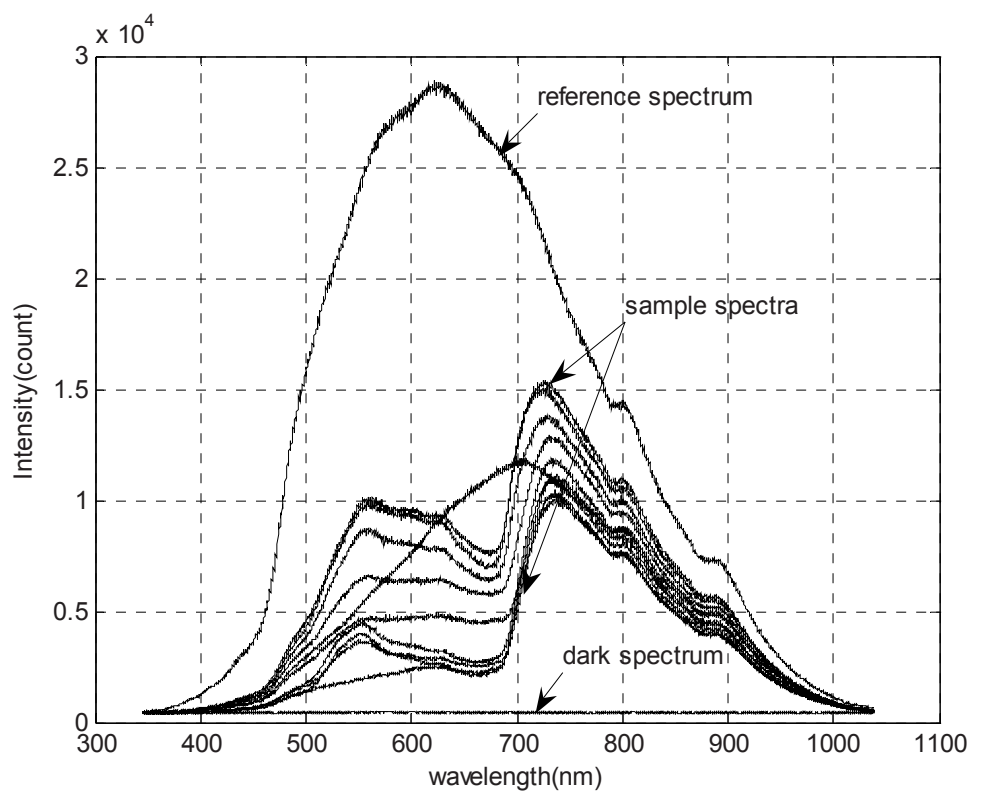

Fig. 1: Reflection spectra of several leave samples

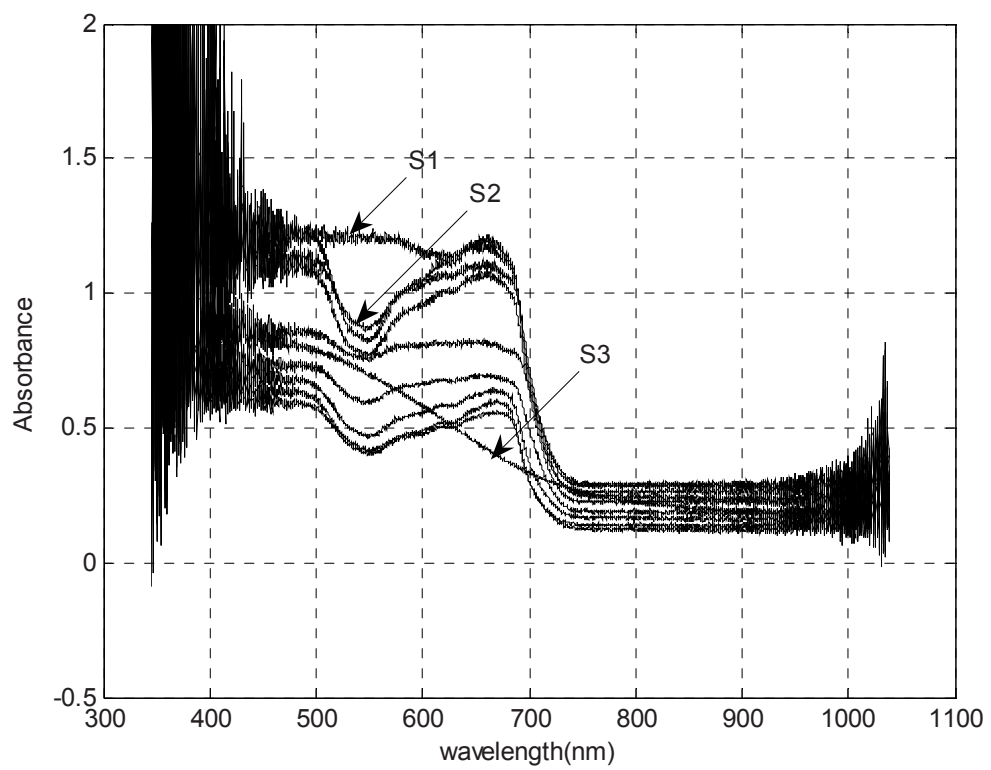

Fig.2: Absorbance spectra of several leave samples

The result is shown in Fig.2. It is obvious that different leaves or different measuring points on one leaf have distinct light absorbance. A purple leaf 
marked by S1 has its SPAD reading of 47.5, another green leaf signed by S2 has the same value. Based on this phenomenon, we found that only the red side from $650 \mathrm{~nm}$ to $750 \mathrm{~nm}$ of each absorbance spectra is close to each other. It gives us an idea that the red side of leaf reflection spectrum can be used for mathematic modeling of leaf chlorophyll prediction. The absorbance spectrum of a yellow dried leaf is shown by S3 in Fig.2. Its spectral absorbance intensity decreases gradually with the wavelength $\lambda$. It has no spectral feature of normal green leaf with high absorbance in red and blue ranges and low absorbance in green and short-wave infrared ranges.

\subsection{Differential absorbance spectrum of leaf sample}

Healthy leaf reflects infrared light and green light but absorbs red light. SPAD-502 meter utilizes two light-emitting diodes (LEDs) with central wavelength of $640 \mathrm{~nm}$ and $950 \mathrm{~nm}$ respectively to measure the relevant absorbance of light. In Fig.2, in the NIR range from $760 \mathrm{~nm}$ to $900 \mathrm{~nm}$, sample absorbance spectra change only in the vertical dimension. To get a uniform baseline for the ease of comparison, a new concept named Differential Absorbance Spectrum (or DAS for short) is adopted to measure the difference between leaf samples.

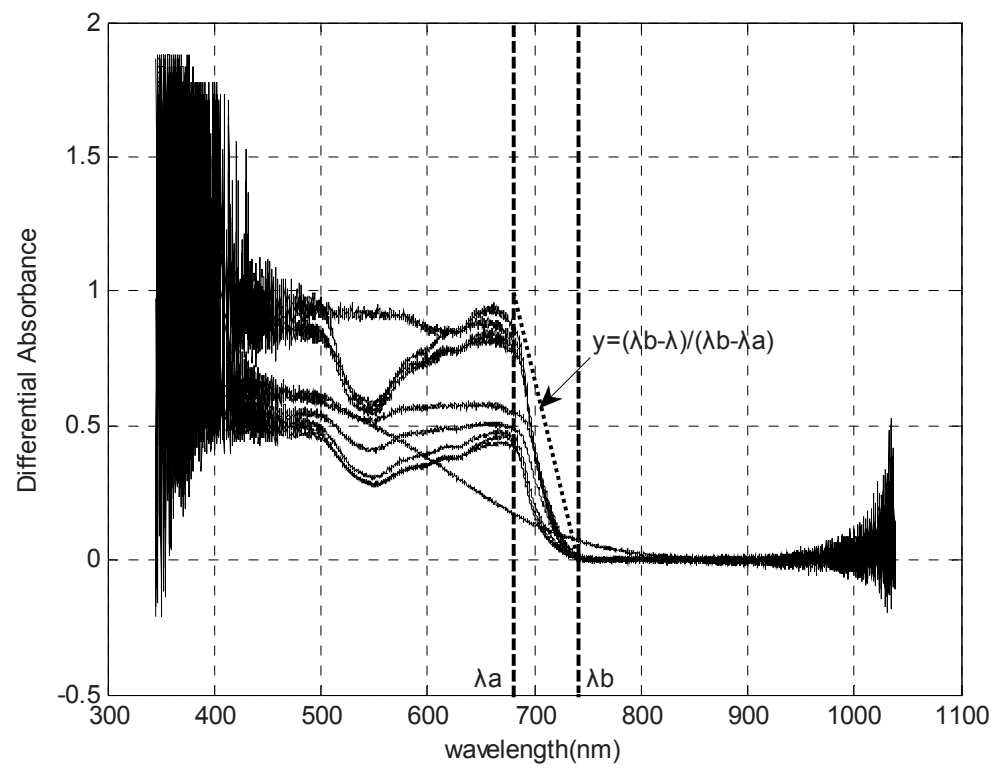

Fig.3: Typical differential absorbance spectra of leaf samples 
Let $D A S(\lambda)$ differential absorbance rate at wavelength $\lambda$, then

$$
D A S(\lambda)=A B S(\lambda)-A V R, \lambda \in(346.01-1038.08 \mathrm{~nm})
$$

where, $A V R$ is the average absorbance from $800 \mathrm{~nm}$ to $900 \mathrm{~nm}$. The typical differential absorbance spectra of leaf samples are shown in Fig.3. Each DAS in the range from $760 \mathrm{~nm}$ to $900 \mathrm{~nm}$ approximates to 0; the DAS in red side range from $650 \mathrm{~nm}$ to $750 \mathrm{~nm}$ decreases monotonously to 0 .

\subsection{Genetic algorithm for leaf chlorophyll levelmodeling}

Based on the observation of the absorbance spectra of leaf samples, we find it feasible to build leaf chlorophyll prediction model in the spectral range of $650-750 \mathrm{~nm}$. Let $\lambda 1$ the lower limit, $\lambda 2$ upper limit. Since SPAD502 meter uses two LEDs as light sources whose spectra have a fixed width with very weak intensity in the upper and lower borders. Thus, we design an adjustable factor of light intensity to modulate the differential absorbance spectra,

$$
\alpha=\frac{\lambda 2-\lambda}{\lambda 2-\lambda 1}
$$

If $\lambda=\lambda 2$, then $\alpha=0$, that would force the DAS at wavelength $\lambda=\lambda 2$ to 0 ; If $\lambda=\lambda 1$, then $\alpha=1$, that would keep the original light intensity unchanged.

Besides, we must consider the influence of leaf thickness in the modeling above. As SPAD-502 meter uses LED's light transmission, leave thickness has been concluded in its measuring result. However, fiber-optic reflection spectrometer can only get the information of leaf surface. According to Beer-Lambert law, the transmission intensity is linearly proportional to the light path so that its absorbance rate is logarithmically proportional to leaf thickness. Thus, the influence factor of leaf thickness can be designed as follows,

$$
\beta=K\left[\log _{10}(\text { thickness })+T\right]
$$

where $K$ and $T$ are the parameters undetermined.

Totally, the prediction equation of leaf chlorophyll level can be entirely written as,

$$
\left.P R E D=K\left[\log _{10} \text { (thickness }\right)+T\right] \sum_{\lambda=\lambda}^{\lambda 2}\left[(A B S(\lambda)-A V R) \frac{\lambda 2-\lambda}{\lambda 2-\lambda 1}\right]
$$

where, $\lambda 1, \lambda 2, K$ and $T$ are the parameters to be determined. Due to the nonlinearity of equation (6), it is difficult to determine the four parameters analytically. In the test, a genetic algorithm (GA) is designed for the parameter optimization.

The procedure of GA is designed as follows, 
Step1: All 70 samples in calibration set $C$ are used to parameter optimization.

Set initial population size 50, each individual with four parameters $\lambda 1, \lambda 2, K$ and $T$. Each parameter is 16 bits wide. The individual fitness function is like,

$$
\text { Fitness }=\sum_{i \in C}|\operatorname{PRED}(\mathrm{i})-\operatorname{REAL}(\mathrm{i})|
$$

where $\operatorname{PRED}(i), \operatorname{REAL}(i)$ is the prediction value and real value of the $i^{\text {th }}$ leaf sample's chlorophyll level. GA termination condition is to run 1000 times.

Step2: Startup GA and generate 50 individuals randomly to form the initial population.

Step3: Calculate the fitness of each individual according to the equation (7). The four parameters with minimal individual fitness in each iterative calculation are kept as the elitist individual. Tournament selection strategy is adopted to renew the population.

Step4: Crossover operation is conducted on the renewed population. The individual number for crossover operation is 20 with the crossover rate of 0.2 . Then mutation operation is performed on each individual with total bits of 10 and mutation rate of 0.01 .

Step5: The iterative number decreases by 1 . If it reaches to 0 , the GA ends, or else, the GA returns to step 3 for next iteration.

Terminally, the optimal result through GA calculation is,

$$
\begin{gathered}
\text { PRED }=0.417\left[\log _{10}(\text { thickness })+3.689\right] \sum_{\lambda=\lambda L}^{\lambda 2}\left[(A B S(\lambda)-A V R) \frac{\lambda 2-\lambda}{\lambda 2-\lambda 1}\right] \\
(\lambda 2=683.24 \mathrm{~nm}, \lambda 1=733.91 \mathrm{~nm})
\end{gathered}
$$

\section{RESULTS}

Leaf chlorophyll statistics regression of calibration set is shown in Fig.4. Part(a) is the result of regression between spectral prediction and meter measurement without leaf thickness adjustment. The regression coefficient $R^{2}=0.2309$, which is very low. Part(b) is the result when the leaf thickness factor is integrated in the calculation. The regression has been improved a lot with $R^{2}=0.8658$. It proves that leaf thickness is an important factor in the modeling of reflective spectroscopy of leaf chlorophyll level. Similarly, the statistics regression of verification set also shows the point (see Fig.5). $R^{2}=0.5675$ (a) without concerns of leaf thickness factor can be improved to $R^{2}=0.9161$ (b) when adding leaf thickness in the modeling. 
Calibration set(Part A. Without concerns of leaf thickness)

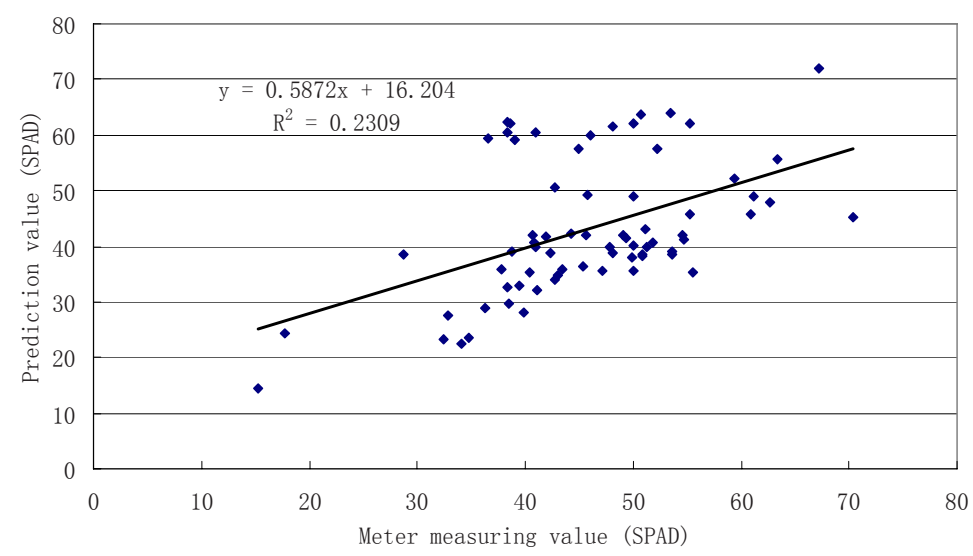

(a)

Calibration set(Part B. With concerns of leaf thickness)

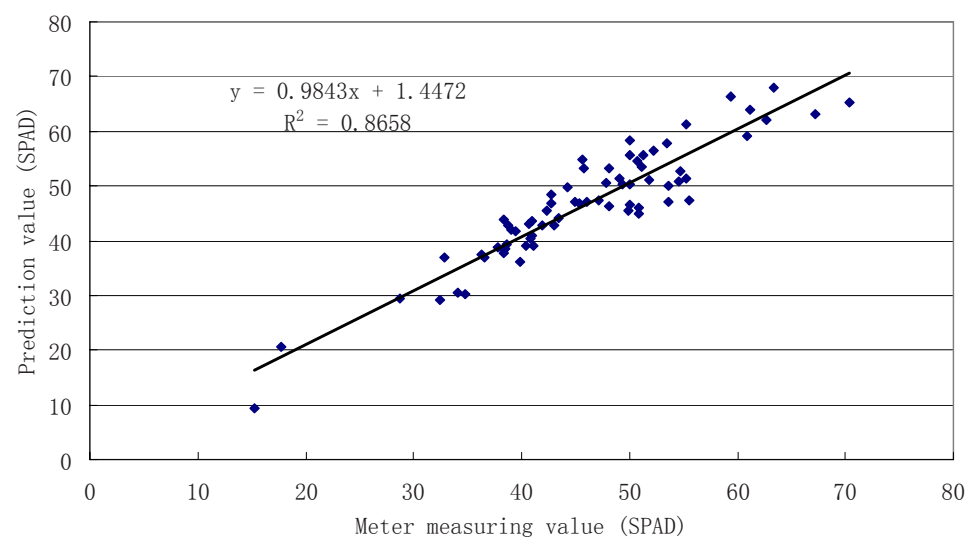

(b)

Fig. 4: Leaf chlorophyll statistics regression of calibration set

\section{CONCLUSION}

In the study, reflection spectroscopy technique was examined in the modeling of leaf chlorophyll level. The result shows that various factors should be put into consideration for the accuracy of spectral modeling. One essential facet is the leaf thickness which has an important effect on the model accuracy. Through observation of absorbance spectral curves, it was found that the spectral range from $650 \mathrm{~nm}$ to $750 \mathrm{~nm}$ is significant for leaf 
chlorophyll model. A genetic algorithm was used to determine the optimal range $683.24-733.91 \mathrm{~nm}$. Besides, an adjustable factor of light intensity was designed to simulate the LED's narrow spectral limitation of SPAD chlorophyll meter to improve the model accuracy. The test proved it reasonable. The prediction model based on reflection spectroscopy has been verified by USB4000 modular spectrometer and could be used as a quantitative model of leaf chlorophyll level.

Verification set(Part A. Without concerns of leaf thickness)

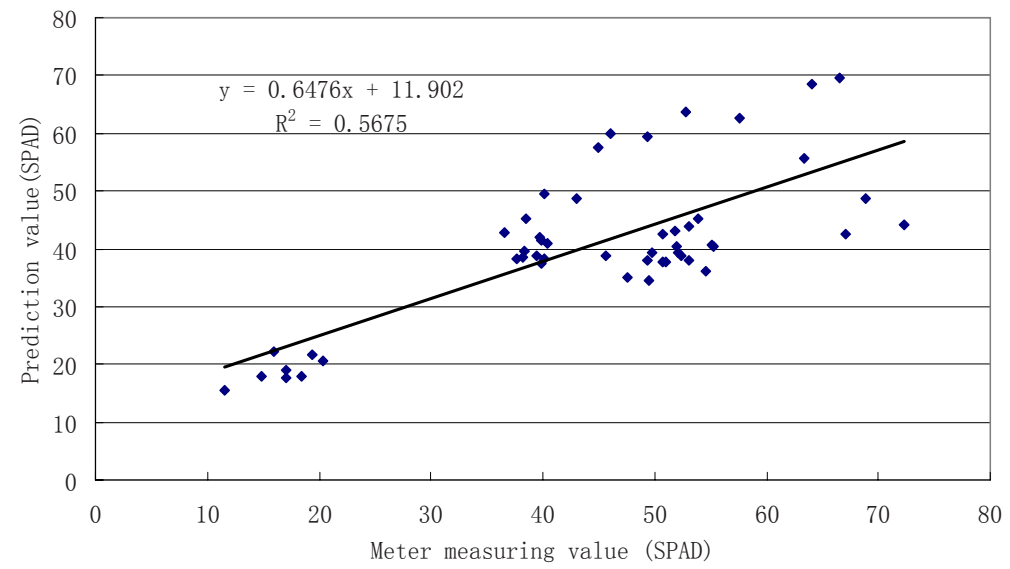

(a)

Verification set(Part B. with concerns of leaf thickness)

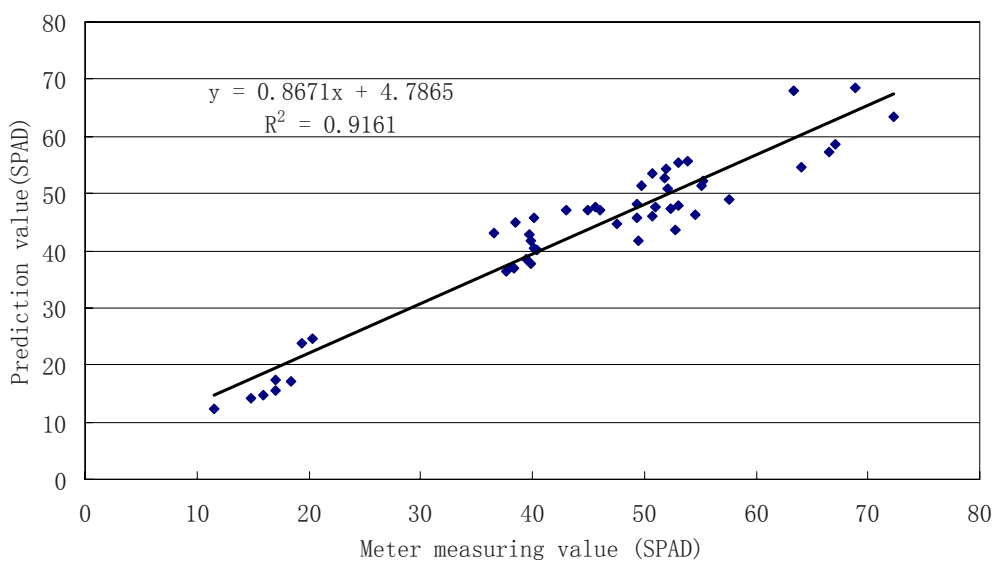

(b)

Fig. 5: Leaf chlorophyll statistics regression of verification set 


\section{ACKNOWLEDGEMENTS}

This research was funded by National Science and Technology Support Program (Project No. 2006BAD10A0403), National High-tech Research and Development Program(863) (2007AA10Z210), National Science Foundation of China (Project No. 30270773), and Key Project of Science and Technology of Ningbo City (Project No.2007C10034).

\section{REFERENCES}

B. O. Hoel, K. A. Solhaud. Effect of Irradiance on Chlorophyll Estimation with the Minolta SPAD-502 Leaf Chlorophyll Meter. Annals of Botany, 1998,82:389-392

E. A. Pinkard, V. Patel, C. Mohammed. Chlorophyll and nitrogen determination for plantation-grown Eucalyptus nitens and E. globulus using a non-destructive meter. Forest Ecology and Management, 2006,223:222-217

F. Wu, L. Wu, F. Xu. Chlorophyll meter to predict nitrogen sidedress requirements for shortseason cotton (Gossypium hirsutum L.). Field crops research. 1998,56:309-314

J. Wu, D. Wang, C. J. Rosen, et al. Comparison of petiole nitrate concentrations, SPAD chlorophyll readings, and QuickBird satellite imagery in detecting nitrogen status of potato canopies. Field Crops research, 2007,101:96-103

Minolta Camera Co. Ltd. Chlorophyll meter SPAD-502 (instruction manual). Radiometric Instruments Divisions, Osaka, Minolta, 1989

Qiu Zhengjun, Song Haiyan, He Yong, Fang Hui. Variation rules of the nitrogen content of the oilseed rape at growth stage using SPAD and visible-NIR. Transactions of the Chinese Society of Agricultural Engineering, 2007,23(7):150-154 (in Chinese)

R. J. Lopez-Bellido, C. E. Shepherd, P. B. Barraclough. Predicting post-anthesis N requirements of bread wheat with a Minolta SPAD meter. European Journal of Agronomy, 2004,20:313-320

S. X. Chang, D. J. Robison. Nondestructive and rapid estimation of hardwood foliar nitrogen status using the SPAD-502 chlorophyll meter. Forest Ecology and Management, 2003,181: 331-338

W. L. Bauerle, D. J. Weston, J. D. Bowden, et al. Leaf absorptance of photosynthetically active radiation in relation to chlorophyll meter estimates among woody plant species. Scientia Horticulturae, 2004,101:169-178

Y. Y. Cho, S. Oh, M. M. Oh, et al. Estimation of individual leaf area, fresh weight, and dry weight of hydroponically grown cucumbers (Cucumis sativus L.) using leaf length, width, and SPAD value. Scientia Horticulturae, 2007,111:330-334

Zhu Zheyan, Bao Yidan, Huang Min, Feng Lei. Study of the relationship between the chlorophyll and the nitrogen content of oilseed rapes. Journal of Zhejiang University (Agriculture and Life Science), 2006,32(2):152 154 (in Chinese) 